\title{
EDUCAÇÃO MATEMATICA E TECNOLOGIAS: UM ESTUDO SOBRE OS RECURSOS EDUCACIONAIS ABERTOS
}

\author{
Maickeon dos Passos ${ }^{1}$ \\ Elisa Netto Zanette ${ }^{2}$
}

\section{INTRODUÇÃO}

O processo de ensino e aprendizagem na sociedade contemporânea apresenta-se como desafiador para os profissionais da educação que investigam e atuam nessa área. Deparam-se nas escolas com as gerações de jovens, nomeados de nativos digitais ou, geração $Y$ e $Z$, segundo Oliveira (2010), com novas formas socioculturais de comunicação e interação em espaço virtual, onde, em geral, o docente é menos familiarizado. Vivem numa sociedade que está interconectada, com novas relações socioeconômicas e vivenciais, bastante diferenciadas em relação às gerações anteriores. Há necessidade de rever os modelos e recursos utilizados nas práxis pedagógicas. Constitui-se, portanto, em desafio para os educadores, que desempenham papel importante no processo de uso desses recursos, como citam Zanette, et al.( 2012).

Parte-se do pressuposto que a utilização de Recursos Educacionais Abertos (REAs), de familiaridades e interesse de uso dos jovens dessas gerações, pode contribuir na melhoria dos processos de sala de aula. Para os professores, as dificuldades e pouca familiaridade com os mesmos e, o pouco tempo disponível para pesquisar e localizar bons recursos nos espaços virtuais, dificultam o seu uso no fazer pedagógico.

Esse cenário foi motivador no direcionamento do presente estudo. Neste contexto, estabeleceu-se como problema da pesquisa: É possível constituir a partir de análise e categorização, um conjunto de REAs relacionados ao processo de ensino e aprendizagem de Matemática na Educação Básica? Definiu-se como objetivo geral, analisar e categorizar os REAs aplicáveis ao processo de ensino e aprendizagem de Matemática na Educação Básica.

\footnotetext{
${ }^{1}$ Universidade do Extremo Sul Catarinense UNESC, acadêmico, maickeon@msn.com

${ }^{2}$ Universidade do Extremo Sul Catarinense UNESC, docente, enz@unesc.net
} 
A pesquisa se estende em ações articuladas ao Grupo de Pesquisa Interdisciplinar em Educação e Cultura Digital (GPIECD) e, com as ações e produções acadêmicas do subprojeto Matemática/PIBID - Programa Institucional de Bolsa de Iniciação à Docência - relacionadas às possibilidades de uso e contribuição das tecnologias digitais na Educação Matemática.

\section{METODOLOGIA}

A pesquisa foi desenvolvida no período de maio de 2015 a fevereiro de 2016. É bibliográfica, com abordagem quali-quantitativa na coleta e análise de dados, cuja finalidade não deve ser a acumulação de fatos, de dados, mas a sua compreensão, como cita Oliveira (1999).

A fundamentação teórica foi baseada em artigos, livros e, em portais educacionais. Baseou-se em autores como Fiorentini (2013), Borba (2001; 2005); Zanette; Mazzuchello (2014); Santos (2013); Nunes et al (2012), entre outros.

Os instrumentos de pesquisa utilizados foram: diário de bordo, formulários com indicadores de qualidade e categorizações. Após a coleta dos dados, estes foram tabulados e analisados e os resultados deverão ser socializados em publicações.

Com base na fundamentação teórica, elaborou-se um instrumento de pesquisa que possibilitou categorizar, analisar e selecionar os RCDs e os REAs, disponíveis na rede Internet. Foram organizados por categorias de softwares educacionais, vídeos, experimentos, áudios e outros. Limitou-se pesquisa dos repositórios e dos REAs de Matemática a portais nacionais, em função da grande quantidade desses portais no cenário mundial, dificultado o processo de seleção e análise no período estabelecido da pesquisa. A análise e validação dos recursos pesquisados foram efetuadas, nesta etapa do estudo, pelo acadêmico pesquisador com o apoio do professor orientador.

\section{A EDUCAÇÃO MATEMÁTICAS E OS RECURSOS EDUCACIONAIS ABERTOS}

A relação da matemática com o cotidiano faz com que o processo de ensino e aprendizagem seja uma pesquisa constante na busca de respostas para entender esse meio social em que alunos e professores estão inseridos 
(JARDINETTI, 2012). A Matemática ainda é vista, por professores e alunos, como uma área de difícil compreensão, segundo Jardinetti (1996). As dificuldades de aprendizagem matemática estão relacionadas à sua complexidade e abstração. $\mathrm{E}$, em geral, suas definições, signos e aplicações não se relacionam com a realidade imediata,

Borba e Penteado (2011) afirmam que, pelo exercício de observação, por exemplo, de formas geométricas que constituem o espaço e, na descrição comparativa de suas diferenças, o aluno elabora uma imagem mental, que possibilita pensar no objeto na sua ausência e se constitui o início de todo o processo de abstração.

Neste sentido, Borba (2001) cita que as atuais tecnologias informáticas, representam uma nova extensão de memória, com diferenças qualitativas em relação às outras tecnologias da inteligência. Possibilitam que a linearidade de raciocínios seja desafiada por modos de pensar, baseados na simulação, na experimentação e em uma nova linguagem que envolve escrita, oralidade, imagens e comunicação instantânea. Nesta perspectiva teórica, as tecnologias atuais reorganizam o pensamento humano na busca da solução de determinado problema matemático.

Para Fiorentini e Lorenzato (2006), o uso das TICs no contexto pedagógico permite aos estudantes estudar temas tradicionais de maneira nova. Borba (2001) evidencia a preocupação com relação ao processo de produção de conhecimento, quando as TICs se inserem no processo de desenvolvimento cognitivo humano. Propõem a concepção teórica de seres humanos com mídias, ou seja, o conhecimento é algo produzido a partir de um coletivo intelectual, composto por humanos e não humanos (mídias oralidade, escrita e informática).

Sobre isso, Moran (1997) afirma que, os recursos educacionais disponíveis na Internet, podem contribuir de forma significativa na melhoria dos processos pedagógicos, incluindo o campo da Matemática. Esses recursos educacionais diversos, aplicáveis ao processo de ensino e aprendizagem, são nomeados de REAs quando se constituem de materiais de ensino, aprendizado e pesquisa, desenvolvidos em suporte digitais, em diferentes mídias.

O termo Recurso Educacional Aberto (REA) tradução de Open Educational Resourses (OER), foi utilizado oficialmente, pela primeira vez, numa conferência em 2002, pela Organização das Nações Unidas para a Educação, 
Ciência e Cultura (UNESCO). O conceito abrangia recursos que, potencializados pelas TICs, possibilitariam a busca, utilização, adaptação e compartilhamentos de conteúdos educacionais para fins não comerciais. Atualmente, engloba animações, simulações, imagens, fragmentos de vídeos, áudios, textos, gráficos, objetos de aprendizagem, ambientes virtuais, módulos de estudo e até cursos (SILVA, 2011).

Os REAs são de domínio público ou estão licenciados de maneira aberta permitindo que sejam utilizados ou adaptados por terceiros (REA-BRASIL, 2011). As licenças mais usuais são: Domínio Público e licença Creative Commons (CC). Atualmente, as licenças Creative Commons (CC), atribuídas pela Creative Commons, uma organização não governamental sem fins lucrativos, esta organizada em seis categorias, que definem as possibilidades de uso: CC-BY atribuição; CC-BY-NC - atribuição e uso não comercial; CC-BY-ND - atribuição e não a obras derivadas; CC-BY-SA - atribuição e partilha pela mesma licença; CCBY-NC-SA - atribuição, uso não comercial e partilha pela mesma licença; e. CC-BYND-NC - atribuição, uso não comercial e não a obras derivadas.

Materiais digitais disponíveis na Web ou em outras tecnologias como CD e DVD sem especificação de licença, indicam que o material tem todos os direitos reservados, o que impede sua reutilização, segundo Santos (2013).

Os REAs estão organizados e publicados em Repositórios de Conteúdos Digitais (RCDs). Para Tarouco, Rodrigues e Schmitt (2013), um repositório digital de recursos e/ou objetos de aprendizagem, se constitui num catálogo digital onde ficam armazenados materiais que possam ser utilizados na educação. Por meio deles é possível localizar e obter materiais para diferentes disciplinas e níveis de ensino. Nos repositórios, ocorre o armazenamento, o registro das versões e de publicação, a busca dos objetos a partir de suas características, o controle de acesso e as avaliações dos objetos. Estes simplificam o acesso aos materiais de qualidade, validados por profissionais da educação.

Para facilitar a localização dos recursos educacionais, em geral, os repositórios tem sistema de busca, como: disciplina, palavra-chave, nível de ensino etc. Essas informações irão contribuir para a localização de um recurso educacional digital mais adequado aos objetivos definidos. A falta dessas especificações dificulta a seleção e uso dos mesmos.

Alguns softwares matemáticos, como o GeoGebra, podem ser classificados de REAs. Em maior ou menor grau, segundo Borba (2001) podem 
promover uma (re)estruturação nas formas de pensar e de agir do aluno; pela intensidade com que ocorrem as interatividades/interações, as simulações, visualização de animações, colaboração entre os pares, entre outros.

No âmbito das tecnologias digitais na educação, os Objetos de Aprendizagem (OA) destacam-se nas pesquisas. De acordo com Santos (2013), são recursos digitais dinâmicos, interativos e reutilizáveis em diferentes ambientes de aprendizagem produzidos a partir de uma base tecnológica. Por este motivo, é importante que, aquilo que for produzido por instituições de ensino, deve ser disponibilizado para outros profissionais da educação. A socialização destes recursos pode trazer melhorias nas práticas pedagógicas de outros professores e também, evitar duplicação de recursos e produções.

Zanette e Mazzuchello (2014) em pesquisas anteriores observaram que, há qualidade e quantidade de materiais disponíveis na rede em repositórios e portais educacionais, que podem ser utilizados na melhoria da educação matemática.

Nas pesquisas efetuadas por Santos (2013) os principais RCDs e REAs disponíveis nestes repositórios são, em geral, publicados com licença Creative Commns e/ou similar e destacam-se pela qualidade e quantidade desses recursos.

\section{RESULTADOS E DISCUSSÕES: ANALISE PRELIMINAR}

A inserção das tecnologias digitais no contexto educacional se justifica pelas constantes transformações sociais e culturais que afetam todos os espaços, incluindo os escolares, que formam e educam as atuais de gerações $Y$ e $Z$ (OLIVEIRA, 2010). Com habilidades e necessidades que as diferenciam das gerações anteriores, provocam o repensar nas ações pedagógicas. Exigem dos professores a reconfiguração de saberes, metodologias e recursos que ampliam o acesso às informações e as possibilidades de fomentar processos cooperativos e colaborativos, adequados a esse perfil de aluno (MORAN, 1997).

As TICs se aproximam da linguagem dos estudantes e faz com que professor e aluno tenham uma relação de aprendizagem colaborativa, onde quem ensina aprende e quem aprende ensina, como afirmam Fiorentini e Lorenzato (2006). O professor assume um papel importante nessa relação, pois precisa ser o mediador desse processo, que poderá promover os estudos contínuos nos alunos. 
Neste contexto, os REAs se constituem como recursos didáticos que podem ser utilizados no fazer pedagógico. Nos repositórios digitais é possível localizar e obter materiais para diferentes disciplinas e níveis de ensino como citam Tarouco, Rodrigues e Schmitt (2013).

Na pesquisa optou-se por analisar e utilizar repositórios de acesso livre, não integrados a Ambientes Virtuais de Aprendizagem. Os RCDs e REAs investigados foram catalogados e categorizados em protocolo de pesquisa com endereço (URL), área de conhecimento, breve descrição e se atende aos objetivos da educação matemática. $\mathrm{Na}$ analise do RCDs e REAs, fundamentou-se em Costa (2007) que propõe indicadores de qualidade organizados em quatro dimensões, integradas: qualidade do conteúdo; design e funcionalidades (facilidade de uso) e pedagogia (potencialidade como ferramenta de ensino-aprendizagem). Para cada dimensão, os indicadores propostos, são:

Identidade: endereço digital; autores; licença; data de criação; última atualização.

Usabilidade: estrutura (páginas bem organizadas; de fácil acesso); navegação e orientação; interface/design; rapidez de acesso; nível de interatividade; espaço de comunicação (contatos, dicas,...).

Conteúdo: tema e adequação às orientações curriculares; abordagem (nível escolar, coerência, lógica e nível linguístico); interdisciplinar; correção do texto (escrito e oral); referências bibliográficas; data de criação e atualização; autoria.

Pedagogia: apresenta potencialidades como ferramenta de ensinoaprendizagem - objetivo, metodologia, recursos, atividades, manual ao professor, entre outros.

Citando como exemplo, o Projeto Folhas é um RCD, mantido pela da Secretaria Estadual da Educação do Paraná. Contém diversos REAs de várias áreas de conhecimento da Educação Básica, incluindo a Matemática. Na análise do RCD, verificou-se que os REAs disponíveis no repositório, foram desenvolvidos de forma interdisciplinar, pelos professores em exercício na rede estadual do Paraná. Tem como objetivo, o aperfeiçoamento dos professores por meio de produção colaborativa de REAs, no formato de folhas. Iniciou na sala de aula onde os professores elaboraram o material didático e outros professores validassem 0 conteúdo. Os materiais didáticos eram, então, transformados em folhas. Depois de validados eram submetidos a dois estágios, o Núcleo Regional de Educação (NRE) 
e o outro pela Secretaria da Educação. Após esse processo era publicado no portal e usado didaticamente pelo professor. Um projeto inovador onde os professores podiam produzir o próprio material didático.

Segundo Fiorentini (2013) as práticas existentes, neste contexto, relatam a proximidade dessas comunidades, professores, acadêmicos e professores de escola pública. Essa prática se torna pertinente na construção de novos conhecimentos. Os formadores e os professores da escola, investigando juntos, elaboram novos modos de ensinar e aprender, construindo parcerias e novas práticas de aprendizagem.

$\mathrm{Na}$ análise do RCD Projeto Folhas a partir das quatro dimensões citadas por Costa (2007), observou-se que indicador identidade está contemplado em todos os REAs de Matemática avaliados. Tem endereço eletrônico http://www.diaadiaeducacao.pr.gov.br, com citação dos autores, tipo de licença, data de criação e última atualização. As licenças são de Domínio Público com observações para citação dos autores quando utilizados.

No indicador usabilidade, os diversos REAs de Matemática tem fácil navegação, com materiais de estudo bem organizados e de fácil acesso. No indicador conteúdo, os REAs são adequados aos temas de estudo para aplicação na Educação Básica. Interdisciplinares, estão organizados com conteúdo matemático fundamentado teoricamente. No indicador pedagogia, apresenta potencialidade como ferramenta de ensino. As orientações de uso da proposta didático-pedagógica estão organizadas de forma a auxiliar o professor na sua utilização em sala de aula.

Procedeu-se de forma similar para a análise dos REAs de outros repositórios avaliados. Estão organizados em tabelas para socialização em espaços virtuais adequados. Os REAs foram categorizados em animações, simulações, áudios, experimentos práticos, hipertextos, imagens, softwares educacionais e vídeos. Foram selecionados somente os REAs aplicáveis a Educação Básica. A seleção e organização de um repositório de REAs, a partir das dimensões citadas por Costa (2007) com os indicadores de qualidade, poderão contribuir nas práxis pedagógicas e na melhoria do processo educativo.

A seleção dos RCDs para o estudo baseou-se na indicação de Santos (2013) com enfoque em experiências brasileiras, algumas em parcerias com instituições internacionais. Os recursos disponíveis, em geral, nesses repositórios são licenciados sobre licença Creative Commns. 
Foram estudados durante a pesquisa, os seguintes RCDs: Bibvirt (Biblioteca Virtual dos Estudantes) e LabVirt vinculado a Escola do Futuro da Universidade de São Paulo (USP); e, Faculdade de Educação da USP (FEUSP); RIVED - Rede Interativa Virtual da Educação; Projeto Folhas; Projeto Condigital; Portal Domínio Público; Portal do Professor; Banco Internacional de Objetos Educacionais; Matemática Multimídia $\left(\mathrm{M}^{3}\right)$; Portal da Secretaria Municipal de Educação de São Paulo, entre outros. Nesses RCD, os professores tem acesso a diversos materiais didáticos que podem ser utilizados no contexto pedagógico.

Ao investiga-los, verificou-se que há grande quantidade de REAs em vários formatos e níveis de ensino. Como afirmam Zanette e Mazzuchello (2014), há qualidade e quantidade de materiais disponíveis na rede em repositórios e portais educacionais, que podem ser utilizados na melhoria da Educação Matemática. Destaca-se, o RCD Banco Internacional de Objetos Educacionais (BIOE). Com mais de vinte mil REAs publicados e um número significativo na área da Matemática, tem autoria de educadores de diversos países. O BIOE disponibiliza muitos recursos didáticos em vários formatos e para todos os níveis de ensino. Criado em 2008, pelo Ministério da Educação e parcerias com outras instituições tem como objetivo, a socialização desses recursos digitais produzidos em diferentes formatos (imagens, vídeo e textos etc...). O BIOE é integrado ao Portal do Professor do Ministério da Educação. Os recursos são de domínio público e parte deles apresenta outras licenças.

Observou-se também, no estudo, que muitas instituições disponibilizam conteúdos didáticos na rede internet, sem especificação da forma de licença de uso. Neste caso, são categorizados como recursos de uso restrito e não são inclusos na categoria de REAs.

\section{CONCLUSÕES PRELIMINARES}

A facilidade de acesso aos diversos RCDs disponíveis na rede Internet, simplificam o processo de acesso e familiarização dos REAs pelo professor e alunos, ampliando a possibilidade de uso dos mesmos. Utilizados com objetivo pedagógico, podem contribuir na melhoria do ensino.

Os REAs representam uma significativa opção para democratizar o acesso ao conhecimento e integram o movimento de educação aberta. A pesquisa 
aponta para uma ampla quantidade de repositórios educacionais na rede Internet, com REAs disponíveis nos formatos de texto, vídeo, áudio, animações, entre outros. Contemplam as diversas áreas de conhecimento, incluindo o campo da Matemática.

A tecnologia utilizada nos repositórios para disponibilização de conteúdos permite acessar via Internet ou fazer download dos recursos digitais em diversos formatos. $\mathrm{Na}$ análise dos mesmos e, a partir dos fundamentos teóricos elaborados, observou-se que a facilidade de acesso aos diversos RCDs disponíveis na rede Internet, simplificam o processo de acesso e familiarização dos REAs pelo professor e alunos, ampliando a possibilidade de uso dos mesmos.

Os REAs investigados têm possibilidade de uso na Matemática visando a melhoria do processo de ensino a aprendizagem. Estes devem ser testados e validados a partir de propostas pedagógicos elaborados pelo professor para sua utilização. Evidenciou-se que, a seleção e organização de REAs de Matemática devem ser constituídas a partir de indicadores de qualidade, de forma a contribuir na melhoria da educação matemática. Propõe-se a continuidade da pesquisa para a ampliação da análise, categorização e validação dos REAs de Matemática, contribuindo na formação de uma base de dados de acesso e consulta livre pelos docentes e discentes da área.

A socialização em espaços virtuais poderá também promover o envolvimento de professores e usuários no processo de análise e avaliação tanto do portal ou blog como dos REA, essencial para fomentar a cultura de compartilhamento, da colaboração, da valorização do trabalho do outro e na visão de uma educação inclusiva e cessível a todos. Sua relevância situa-se na ampliação dos espaços de acesso democrático a educação, a materiais de qualidade e permite inovação metodológica que atende ao perfil das novas gerações.

\section{REFERÊNCIAS}

BORBA, M. C.; PENTEADO, M. G. Informática e Educação Matemática. Belo Horizonte, MG: Autêntica, 2001.

BORBA, M. C. Coletivos Seres-Humanos-com-Mídias e a Produção de Matemática. In: Anais do Simpósio Brasileiro de Psicologia da Educação Matemática, 1., 2001, Curitiba. 
Costa, F. A aprendizagem como critério de avaliação de conteúdos educativos on-line. Caderno SACAUSEF - Sistema de Avaliação, Certificação e Apoio à Utilização de Software para a Educação e a Formação, Número 2, Ministério da Educação, 45-54. Disponível em:< http://nautilus.fis.uc.pt/personal/jcpaiva/disc/me/rec/03/04/01/texto3.pdf> Acesso em: 04 Maio 2015.

FIORENTINI, D. A Investigação em Educação Matemática desde a perspectiva acadêmica e profissional: desafios e possibilidades de aproximação. In: Portal de Revistas Acadêmicas. 2013. Año 8. Número 11. pp 61-82. Costa Rica. ISSN impresso 1659-2573. Disponível em:

http://revistas.ucr.ac.cr/index.php/cifem/article/view/14711. Acesso: 10 Jul 2015.

FIORENTINI, D.; LORENZATO, S. Investigação em educação matemática: percursos teóricos e metodológicos. Campinas, SP: Autores Associados, 2006.

JARDINETTI, J.R.B. O saber escolar como parte das formas mais desenvolvida de saber: A questão cultural na educação Matemática. 2012. In: EMP Educação Matemática e Pesquisa. PUC/SP. V.14, n.1, 2012. Disponível em: <http://revistas.pucsp.br/index.php/emp/article/view/7583>. Acesso: 03 jul 2015.

JARDINETTI, José Roberto Boettger. Abstrato e concreto no Ensino da Matemática: algumas reflexões. In: Bolema. Rio Claro, SP: UNESP, ano 11, n.12, 1996. p. 45-57.

MORAN, J.M. Como utilizar a internet na educação. Revista Ciência da Informação. V.26, n.2, maio/agosto 1997. p. 146-153.

OLIVEIRA, S. Geração Y: O nascimento de uma nova versão de líderes. SP: Integrare Editora. 2010.

OLIVEIRA, S. L. Trabalho de Metodologia Científica. São Paulo: Pioneira, 1999.

REA-BRASIL. O que é REA? . 2011. Disponível em <http://rea.net.br/site/o-que-erea/>. Acesso em 05 Jul 2015.

SANTOS, A.I. Recursos Educacionais Abertos no Brasil: [livro eletrônico] : O Estado da Arte, Desafios e Perspectivas para o Desenvolvimento e Inovação; [tradução DB Comunicação]. São Paulo : Comite Gestor da Internet no Brasil, 2013. 1,6Mb; PDF. Disponível em <http://www.cetic.br/publicacoes/2012/rea-andreiainamorato.pdf>. Acesso em: 05 Maio 2015.

SILVA, R.S. Objetos de Aprendizagem para Educação a Distância. São Paulo: Novatec Editora, 2011. 
TAROUCO, L.M.R.; RODRIGUES, A.P.; SCHMITT, M.A.R. Integração do MOODLE com repositórios abertos. Revista Perspectivas em Ciência da Informação, v.18, ano 1. Belo Horizonte Jan./Mar. 2013. Disponível em:

http://www.scielo.br/scielo.php?pid=S1413-99362013000100006\&script=sci_arttext. Acesso em: 10 Jun 2015.

ZANETTE, E.N.; GIACOMAZZO, G.F.; FIUZA, P.J. (Org.). Tecnologias e Inovações nas Práticas Pedagógicas: Trajetórias e Experiências. $1^{\mathrm{a}} \mathrm{ed}$. Jundiaí/SP: Paco Editora. 2012.

ZANETTE, E.N.; MAZZUCHELLO, S. B.; Os recursos educacionais abertos digitais (READs) e processo de ensino e aprendizagem de matemática. Congresso Ibero Americano de Humanidades e Educação. Criciúma/SC: Universidade do Extremo Sul Catarinense UNESC, 2014. 